\title{
Extensive Air Showers and Cosmic Ray Physics above $10^{17} \mathrm{eV}$
}

\author{
Mario Bertaina ${ }^{1,2, a}$ \\ ${ }^{1}$ Dipartimento di Fisica, Università degli Studi di Torino - Italy \\ ${ }^{2}$ Istituto Nazionale di Fisica Nucleare - Sezione di Torino - Italy
}

\begin{abstract}
Cosmic Rays above $10^{17} \mathrm{eV}$ allow studying hadronic interactions at energies that can not be attained at accelerators yet. At the same time hadronic interaction models have to be applied to the cosmic-ray induced air-shower cascades in atmosphere to infer the nature of cosmic rays. The reliability of air-shower simulations has become the source of one of the largest systematic uncertainty in the interpretation of cosmic-ray data due to the uncertainties in modeling the hadronic interaction driving the air-shower development. This paper summarises in the first part the recent results on the cosmic ray energy spectrum, composition and anisotropy from the knee region to the GZK cutoff $[1,2]$ of the spectrum by means of ground-based experiments. Most of the information reported in this contribution is taken from [3-5]. Aspects interconnecting cosmic ray and particle physics are reviewed in the second part of the paper.
\end{abstract}

\section{Introduction}

The cosmic ray energy spectrum above $10^{14} \mathrm{eV}$ has a power-law like behaviour ( $\propto E^{-\gamma}$, with $\gamma \sim 2.7$ ) with features which are known as the 'knee' at $3-4 \times 10^{15} \mathrm{eV}$, where the spectrum steepens to $\gamma \sim 3.0$, the 'ankle' at $2-8 \times 10^{18} \mathrm{eV}$, which is characterised by a flattening of the spectrum by roughly the same change of the spectral index, i.e. back to $\gamma \sim 2.7$, and the GZK cut-off around $5 \times 10^{19} \mathrm{eV}$.

The shape and composition of the primary spectrum as well as the large-scale anisotropy in the arrival direction of cosmic rays are key elements to understand the origin, acceleration and propagation of the Galactic radiation. The paradigm of the origin of Galactic cosmic rays (CR) are supernovae, as their shock waves can provide the required power to explain the intensity of the CR radiation at least up to $10^{15} \mathrm{eV}$ [6]. This paradigm has been recently confirmed well below the knee by the observations of AGILE [7] and FERMI satellites [8]. However, the possibility for supernovae to accelerate CRs at energies above $10^{15} \mathrm{eV}$ is quite challenging, therefore, different populations of sources have been envisaged as responsible for the radiation in galactic and extragalactic energy ranges $[9,10]$. Those sources would be subject to a rigidity cutoff in the maximum energy at which the various elements are accelerated, as proposed originally by Peters [11]. In this approach, the knee at $\sim 4 \times 10^{15} \mathrm{eV}$ would represent the end of the spectrum of CRs accelerated by supernova remnants in the Milky Way and the ankle at $\sim 4 \times 10^{18} \mathrm{eV}$ the transition to particles from extragalactic sources. However, the ankle structure could be explained also in a completely different way, such as a consequence of the physical process of pair production by protons during propagation through the cosmic microwave

\footnotetext{
a e-mail: bertaina@to.infn.it
} 
background radiation [12]. In this case, the Galactic-extragalactic transition occurs below $10^{18} \mathrm{eV}$. Around $\sim 5 \times 10^{19} \mathrm{eV}$ another break is observed. Its position is compatible with the GZK cutoff, however, it could be explained also as the limit in the UHECRs acceleration by the sources.

A refined study of the CR primary spectrum and composition is, therefore, extremely important to address the above questions. As acceleration and propagation mechanisms in magnetic fields would lead to the same rigidity dependence, the study of large scale anisotropies in the arrival direction could provide relevant information to distinguish source and propagation effects.

The direct study of CRs by means of satellites or balloon-borne detectors is performed only at energies below $10^{15} \mathrm{eV}$. Close to the knee, the flux becomes of the order of 1 particle $\mathrm{m}^{-2} \mathrm{sr}^{-1} \mathrm{yr}^{-1}$. This fact prevents the possibility of a direct observation of its structure by currently planned satellites or balloon experiments. Indeed, at least hundred of events above the knee are necessary to determine its existence with enough significance. Around the knee and at higher energies, CRs are studied by means of large arrays located at ground that measure the secondary particles produced by the primary CR cascading in the atmosphere, the so-called Extensive Air Showers (EAS). Typically, the energy is proportional to the total number of secondaries sampled at ground, while the composition is inferred either through a multi-component measurement, such as the electromagnetic and muonic components, or through the measurement of the emitted light (Cherenkov or fluorescence lights) along the longitudinal development of the shower. Despite the fact that shower arrays allow one to collect high statistics, the interpretation of the results is based on the comparison with expectations from simulation describing the EAS development in atmosphere, which are at some level inaccurate. This introduces a systematic uncertainty on the results, especially on the mass composition.

The $\mathrm{TeV}$ region allows some partial overlap between direct and indirect measurements. Several techniques have been employed recently on ground detectors that are sensitive to specific components of the CR radiation to overcome those uncertainties [13]. Among them, it is worth mentioning the measurement of the light component ( $\mathrm{p}$ alone, or $\mathrm{p}+\mathrm{He}$ ) using hadron calorimeters [14], or Cherenkov light measurements in coincidence with TeV muons [15], and RPC counters at high altitude [16]. Those results are in quite good agreement with measurements by CREAM [17] balloon. In particular, the ARGO results allow one to cross-check the fluxes on an extended energy range (5-250 TeV). These results show that, when indirect measurements have the opportunity of selecting almost pure beams, their findings are in reasonable agreement with direct ones and confirm a fair representation of the EAS development in the atmosphere by simulation codes such as CORSIKA [18].

\section{The knee region up to the ankle}

The 'knee' is a distinct feature of the all-particle CR energy spectrum at $\sim 4 \times 10^{15} \mathrm{eV}$, where the power index suddenly changes from $\gamma \sim-2.7$ to $\gamma \sim-3.1$. Since its discovery the origin of this feature is still under debate. From the experimental point of view, measurements indicate that such a break is observed in the hadronic, muonic, and electromagnetic components [19-22], as well as in Cherenkov light [23]. These results give a clear indication that the knee is a peculiarity of the primary spectrum, disfavouring a hypothesis based on changes of the interaction characteristics of the primaries with air nuclei. This conclusion has been reinforced by the first comparisons of the predictions from hadronic models and LHC data [24].

Several experimental results associate the knee with the bending of the light component, and are compatible with a rigidity dependent cut-off $[20,21]$. Unfortunately, the flux of the different components vary significantly depending on the interaction model used to interpret the data [21]. The recent results from the ARGO experiment, however, are in tension with this interpretation as they indicate that the proton component bends already around $1 \mathrm{PeV}$ [25]. Nevertheless, if the interpretation that 


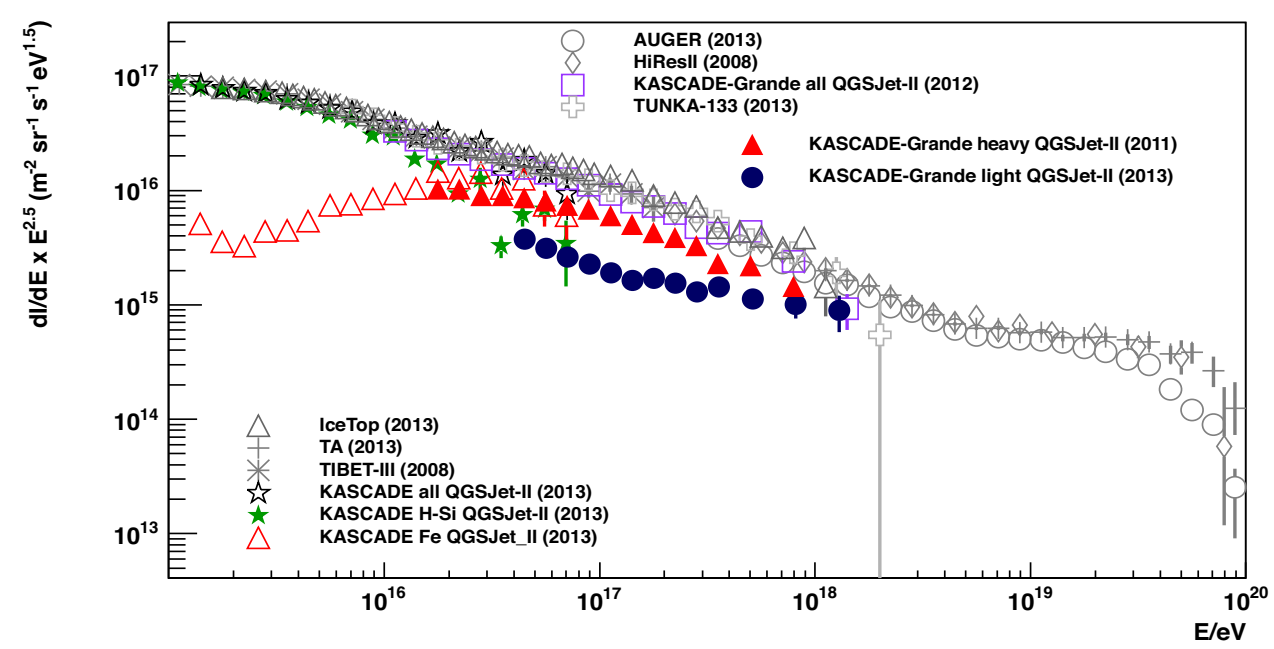

Figure 1. The measurement of the CR spectrum by EAS experiments from the knee till the end of the spectrum. The plot is taken from [3].

the main knee is due to the bending of the light component is correct, the heavy one should show a similar bending in the energy range $5 \times 10^{16}-10^{17} \mathrm{eV}$. This is indeed the experimental finding of KASCADE-Grande [26] confirmed by TUNKA-133 [27] and IceTop [28] experiments.

In detail, the measured all-particle energy spectrum by KASCADE-Grande exhibits a less pronounced but still clear deviation from a single power law between the knee and the ankle, with a spectral hardening at $\sim 2 \times 10^{16} \mathrm{eV}$ and a steepening at $\sim 10^{17} \mathrm{eV}$ [29]. The average mass composition gets heavier after the knee till $\sim 10^{17} \mathrm{eV}$, where a bending of the heavy component is observed [30]. An indication of a hardening of the light component just above $10^{17} \mathrm{eV}$ has been measured as well [31]. The flux of heavy and light components depends on the hadronic interaction model used to interpret the experimental data [32] as previously mentioned. The above results are summarised on Fig. 1.

Fig. 2 compares the results of several experiments in terms of $\langle\ln A\rangle$, as it is often reported in literature to describe the evolution of the composition as a function of energy. Only QGSJet-II model is considered. A detailed description including the role of interaction models in composition is reported in [33]. Use of different models introduces a shift in the average mass comparable to the dispersion of the data in Fig. 2. Despite the large uncertainty in the absolute composition, a common general trend is visible. Composition gets heavier through the knee region and becomes lighter approaching the ankle. The solid line in the plot is used as a guidance line to show how the average $<\ln A>$ of data in the plot evolves with energy. This result is compatible with the concept that the galactic component of the cosmic ray radiation reaches an end following a rigidity cut-off at $\sim 10^{17} \mathrm{eV}$ and that the ankle indicates a transition to a population dominated by extra-galactic sources.

The search for anisotropies in the arrival direction of CR can provide relevant information to distinguish source and propagation effects. The anisotropy varies with energy but the topological structure remains the same till $\sim 10^{14} \mathrm{eV}$ where it has an abrupt change as pointed out by IceCube [34]. Such a change is confirmed till knee energies. This result seems to be inconsistent with the amplitude and phase expected according to the Compton-Getting prediction due to the relative motion of the Earth in the Galaxy. KASCADE-Grande published recently [35] an update on the anisotropy study based on 


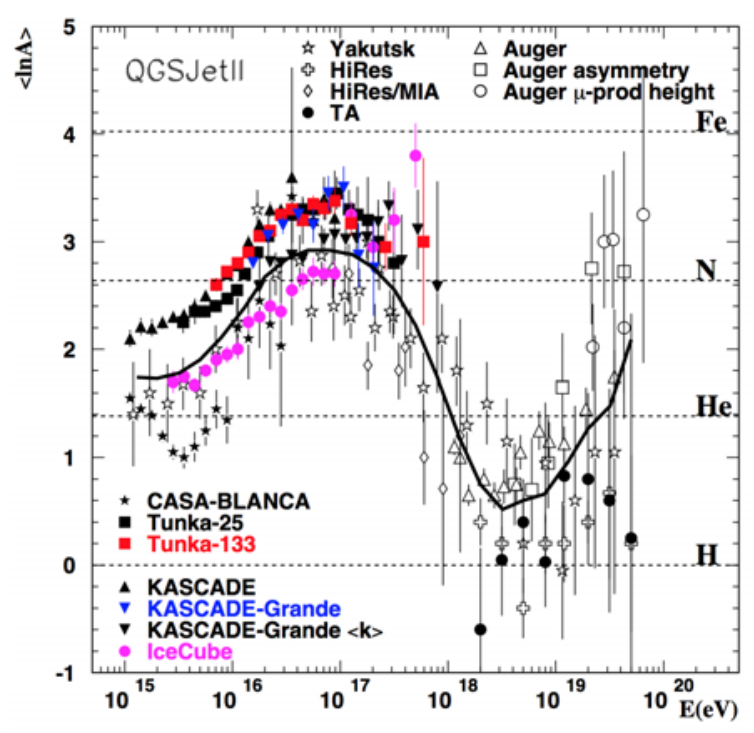

Figure 2. Average logarithmic mass of CRs as a function of energy derived from $X_{\max }$ and particle detector measurements using QGSJet-II interaction model. Most of the data are taken from [33] and references therein. The solid line is drawn as a guidance and is obtained by averaging, in each energy bin, the values of $<\ln A>$ reported in the figure. The plot is taken from [3].

the East-West method [36]. By investigating the variation of the amplitude as a function of energy it was found that the amplitudes were not significant, however, the phases were in almost all energy bins centred around $250 \pm 25$ degrees. This is interesting in itself because it points towards the Galactic Center. Moreover, it agrees inside the statistical uncertainties with the results of the anisotropy studies of the Pierre Auger Observatory (PAO) [37] in the range $3 \times 10^{17}-10^{18} \mathrm{eV}$.

\section{From the ankle to the end of the spectrum}

Fig. 1 shows that the features at the Ultra High Energies (UHE), the ankle and the cutoff, have been established beyond doubt. The spectral slopes before and after the ankle have been measured and agree between PAO [38] and Telescope Array (TA) [39]. The positions of the ankle also agree within the quoted errors, and are compatible with the existing model(s). The parameters of the break at the highest energies are known less accurately. There seems to be some discrepancy concerning the shape of the spectrum around the break; however more statistics is needed for a firm conclusion. The position of the break is compatible with the GZK cutoff for protons in TA, but the PAO spectrum fits better the case of a limit in the UHECRs acceleration by the sources.

As far as the mass composition is concerned, the situation is less definite [33]. While PAO sees a change in the composition towards a heavier one at the highest energies, the TA observes no such a trend and is compatible with a pure proton composition. This difference in the data has profound consequences: the Auger data suggest that we see the maximum energy of sources, similarly to what is observed at the knee in the cosmic ray spectrum, while the TA data suggest we observe the GZK effect. Moreover, the different compositions in the GZK- and maximum-energy scenario will affect 
the level of anisotropies expected to be seen in the data. It should be noticed, however, that a recent analysis of the joint working group on composition of the PAO and TA collaborations, seem to reconcile the two results obtained independently by PAO and TA experiment [40]. In this analysis an adequate comparison was achieved by taking into account that the $\left\langle X_{\max }>\right.$ published by PAO are corrected for detector effects, whereas those published by TA includes detector effects. From the preliminary comparison the authors conclude that the data of the two observatories are in good agreement. Therefore, the apparent differences seem to be related to the different hadronic model used to compare the data and to the role of detector effects which doesn't allow a direct comparison between the two results.

Despite the major advances in the understanding of UHECRs nature, the current experiments face the limit in the statistics they can accumulate due to the extremely low flux of 1 particle $\mathrm{km}^{-2}$ year $^{-1}$ above $5 \times 10^{19} \mathrm{eV}$. For this reason future space-based observatories, such as JEM-EUSO, that reach much higher and uniform exposures [41], could help in sheding light on the mystery of the most energetic radiation of the Universe.

\section{The interconnection between cosmic ray and particle physics}

A fundamental ingredient of the CORSIKA simulation is the hadronic interaction model which generates the hadronic cascade at the origin of the electromagnetic one. Since 2009, the Large Hadron Collider (LHC) provides a lot of very precise data which have been used to improve the hadronic models used for air shower simulations, such as EPOS [42] and QGSJetII [43], giving birth to the most updated versions: EPOS-LHC and QGSJetII-04. References [44] and [45] provide, respectively, the most updated review on the measurements at LHC and their relevance for UHECRs and a review on the cosmic ray interaction models.

Remarkably, interaction models employed in air shower simulations provide a reasonable prediction of global observables (multiplicities, $p_{T}$-distributions, forward and transverse energy flow, etc.), in some cases even better than typical tunes of HEP models, such as PYTHIA or PHOJET [46]. The cross-section is particularly important for the EAS development and the depth of shower maximum. The proton-proton scattering total cross-section measured by TOTEM [47] at $\sqrt{s}=7 \mathrm{TeV}$ allowed to reduce to $20 \mathrm{gr} / \mathrm{cm}^{2}$ the difference in $X_{\max }$ position of the two models, which is comparable to the systematic uncertainties in the measurement by experiments. Detecting UHECRs enables to measure the proton-air inelastic cross section $\sigma_{\mathrm{p} \text {-air }}^{\text {inel }}$ at energies that can not be reached with particle acceleerators. The proton-proton cross section $\sigma_{\mathrm{p}-\mathrm{p}}$ is subsequently inferred from the proton-air cross section at these energies. UHECRs experiments have been reported results on the proton-air inelastic cross section till $\sqrt{s} \sim 100 \mathrm{TeV}$. A recent review on this topic is reported in [48]. The muon number depends on the ratio between particles producing hadronic sub-showers and the total number of particles. LHC data allow constraining the (anti)baryon and strangeness production at mid rapidity as well as the forward production of $\pi^{0}$ in fixed target experiments [49]. Taking into account these data, EPOSLHC and QGSJetII-04 provide now better results [50] compared to the previous versions (see Fig. 3). Despite improvements obtained using the updated version of the interaction models, shortcomings still exist. As an example PAO estimate the muon number at ground and the muon production depth using measurements of the longitudinal, lateral and temporal disstribution of particles in the EAS. The results, obtained at about $140 \mathrm{TeV}$ center-of-mass energy for proton primaries, are compared to the predictions of LHC-tuned hadronic interaction models for different primary masses. The models exhibit a deficit in the muon content [52]. 

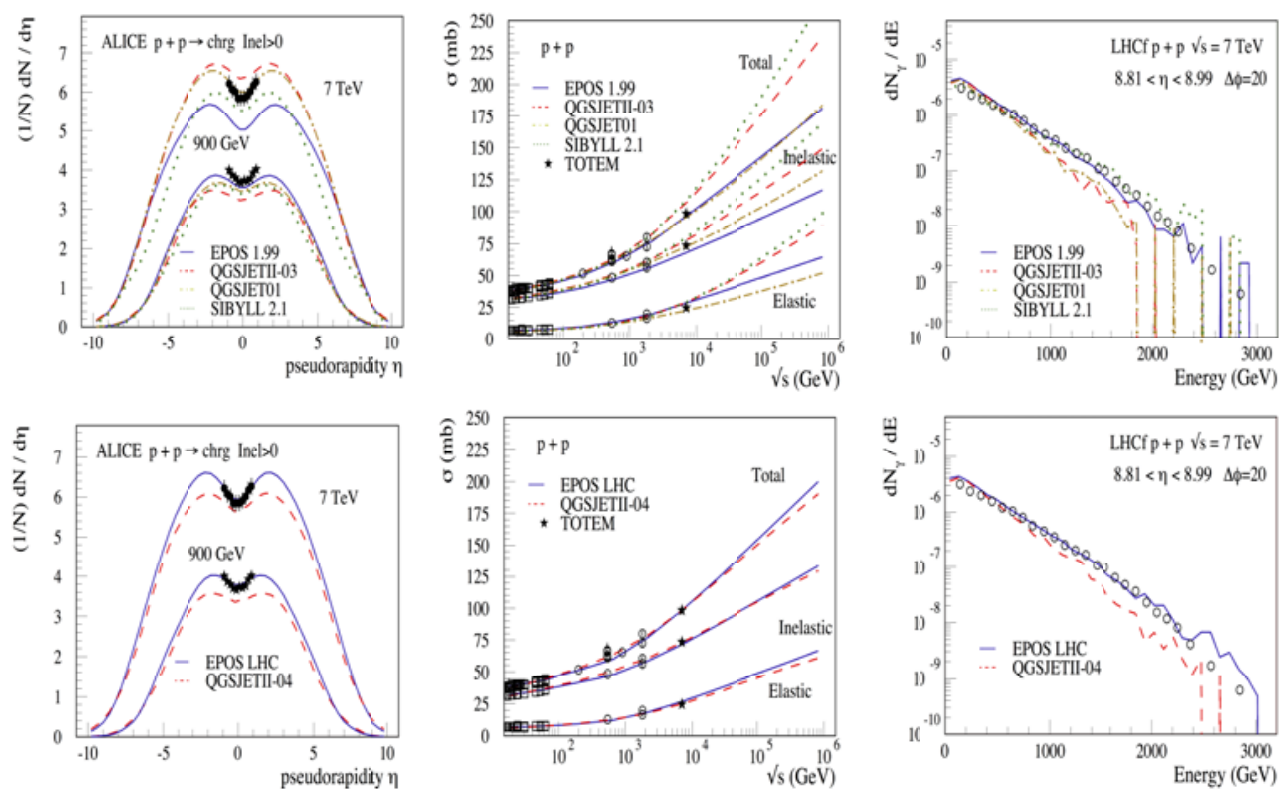

Figure 3. Examples of tuning interaction models to LHC data. Top (bottom) panel shows a comparison between old (new) versions of hadronic interaction models used in CR science and LHC data. Left column refers to pseudorapidities, central one to cross-sections and right one to distributions of particle energy (plot from [51]).

\section{Conclusions}

A review of the current understanding of cosmic ray data at different energies has been presented. The interpretation of $\mathrm{CR}$ data requires the knowledge of the physics of hadronic interactions in atmosphere, but at the same time provides a means to cross-check the validity of the physics principles embedded in the models. Hadronic interaction models do a fairly well job not only in the interpretation of EAS cascades in atmosphere but also of LHC data. However, shortcomings exist. LHC data are extremely helpful in fine tuning the models and give solid bases for the extrapolation at high energies. CR remain the sole mean to test hadronic interactions at energies well beyond those reachable with colliders. CRs and accelerator data provide an excellent mix of information to understand the physics of interactions.

\section{References}

[1] Greisen K., Phys. Lett. 16148 (1966).

[2] Zatsepin G.T. and Kuz'min V.A., JETP 478 (1966).

[3] Bertaina M., Comptes Rendus Phys. 15/4 300 (2014).

[4] Kampert K.-H. and Tinyakov P., Comptes Rendus Phys. 15/4 318 (2014).

[5] Bertaina M., Il Nuovo Cimento 38 C 29 (2015).

[6] Blasi P., Astron. Astrophys. Rev. 211174 (2013).

[7] Giuliani A. et al. (AGILE Coll.), Astrophys. J. 742 L30 (2011).

[8] Ackermann M. et al. (FERMI-LAT Coll.), Science 339807 (2013). 
[9] Hillas M., J. Phys. G, Nucl. Part. Phys. 31 R95 (2005).

[10] Gaisser T, Stanev T. and Tilav S., arXiv: 1303.3365v1 (2013).

[11] Peters B., Il Nuovo Cimento 22800 (1961).

[12] Berezinsky V., Gazizov A. and Grigorieva S., Phys. Rev. D 74043005 (2006).

[13] Bertaina M. et al., J. Phys. Soc. Jpn. Suppl. A 782010 (2009).

[14] Aglietta M. et al. (EAS-TOP Coll.), Astrop. Phys. 19329 (2003).

[15] Aglietta M. et al. (EAS-TOP and MACRO Coll.), Astrop. Phys. 21223 (2004).

[16] Bartoli B. et al. (ARGO-YBJ Coll.), Phys. Rev. D 85092005 (2012).

[17] Yoon Y.S. et al. (CREAM Coll.), Astrophys. J. 728122 (2011).

[18] Heck D., Report FZKA 6019 (1998).

[19] Aglietta M. et al. (EAS-TOP Coll.), Astrop. Phys. 101 (1999).

[20] Aglietta M. et al. (EAS-TOP Coll.), Astrop. Phys. 21583 (2004).

[21] Antoni T. et al. (KASCADE Coll.), Astrop. Phys. 241 (2005).

[22] Aglietta M. et al. (EAS-TOP and MACRO Coll.), Astrop. Phys. 20641 (2004).

[23] Budnev N. et al. (TUNKA Coll.), Astrop. Phys. 50-52 18 (2013).

[24] Ostapchenko S., Prog. Theor. Phys. Suppl. 193204 (2012).

[25] Bartoli B. et al. (ARGO-YBJ Coll.), Phys. Rev. D 92092005 (2015).

[26] Apel W.D. et al. (KASCADE-Grande Coll.), Nucl. Instr. Meth. A 620202 (2010).

[27] Prosin V.V. et al. (TUNKA-133 Coll.), Nucl. Instr. Meth. A 75694 (2014).

[28] Aartsen M.G. et al. (IceTop Coll.), Phys. Rev. D 88042004 (2013).

[29] Apel W.D. et al. (KASCADE-Grande Coll.), Astrop. Phys. 36183 (2012).

[30] Apel W.D. et al. (KASCADE-Grande Coll.), Phys. Rev. Lett. 107171104 (2011).

[31] Apel W.D. et al. (KASCADE-Grande Coll.), Phys. Rev. D 87 081101(R) (2013).

[32] Apel W.D. et al. (KASCADE-Grande Coll.), Adv. Space Res. 531456 (2014).

[33] Kampert K.-H. and Unger M., Astrop. Phys. 35660 (2012).

[34] Abbasi R. et al. (ICECUBE Coll.), Astrophys. J. Lett. 718 L194 (2010).

[35] Chiavassa. et al. (KASCADE-Grande Coll.), Proc. 33 ${ }^{\text {rd }}$ ICRC, \#0093 (2013).

[36] Bonino R. et al., Astrophys. J. 73867 (2011).

[37] Abreu P. et al. (Pierre Auger Coll.), Astrop. Phys. 34627 (2011).

[38] Abraham J. et al. (Pierre Auger Coll.), Phys. Rev. Lett. 101061101 (2008).

[39] Abu-Zayyad T. et al. (Telescope Array Coll.), Astrophys. J. 768 L1 (2013).

[40] Unger M. et al. (PAO and TA Colls.), Proc. $34^{\text {th }}$ ICRC, PoS(ICRC2015) \#307 (2015).

[41] Adams Jr. J.H. et al. (JEM-EUSO Coll.), Astrop. Phys. 4476 (2013).

[42] Werner K., Liu F.-M. and Pierog T., Phys. Rev. C 74044902 (2006).

[43] Ostapchenko S., Phys. Rev. D 74014026 (2006).

[44] Ulrich R., These Proceedings (2015).

[45] Ostapchenko S., These Proceedings (2015).

[46] d'Denterria D. et al., Astrop. Phys. 3598 (2011).

[47] Antchev G. et al. (TOTEM Coll.), Europhys. Lett. 9621002 (2011).

[48] Abbasi R., These Proceedings (2015).

[49] Adriani O. et al. (LHCf Coll.), Phys. Rev. C 89065209 (2014).

[50] Pierog T. and Heck D., Proc. $33^{\text {rd }}$ ICRC, \#0163 (2013).

[51] Engel R., Proc. $34^{\text {th }}$ ICRC, Invited talk (2015).

[52] Veberic D. (Pierre Auger Coll.), These Proceedings (2015). 